EPJ manuscript No.

(will be inserted by the editor)

\title{
Kaon-nucleon scattering lengths from kaonic deuterium experiments
}

\author{
Ulf-G. Meißner ${ }^{1,2}$, Udit Raha ${ }^{1}$, and Akaki Rusetsky ${ }^{1,3}$ \\ 1 Universität Bonn, Helmholtz-Institut für Strahlen- und Kernphysik (Theorie), \\ Nußallee 14-16, D-53115 Bonn, Germany \\ 2 Forschungszentrum Jülich, Institut für Kenphysik (Theorie), D-52425 Jülich, Germany \\ 3 On leave of absence from: High Energy Physics Institute, Tbilisi State University, \\ University St. 9, 380086 Tbilisi, Georgia
}

Received: date / Revised version: date

\begin{abstract}
The extraction of the S-wave kaon-nucleon scattering lengths $a_{0}$ and $a_{1}$ from a combined analysis of existing kaonic hydrogen and synthetic deuterium data has been carried out within the framework of a low-energy effective field theory. It turns out that with the present DEAR central values for the kaonic hydrogen ground-state energy and width, a solution for $a_{0}$ and $a_{1}$ exists only in a restricted domain of input values for the kaon-deuteron scattering length. Consequently, measuring this scattering length imposes stringent constraints on the theoretical description of the kaon-deuteron interactions at low energies.
\end{abstract}

PACS. 36.10.Gv - 12.39.Fe - 13.75.Cs $-13.75 . \mathrm{Jz}$

\section{Introduction}

Recently, the DEAR collaboration at LNF-INFN has per' formed a measurement of the energy level shift and width of the kaonic hydrogen ground state [1] with a considerably better accuracy than the earlier KpX experiment at KEK [2]. The preliminary result of DEAR is

$$
\begin{aligned}
\epsilon_{1 s} & =193 \pm 37 \text { (stat) } \pm 6 \text { (syst) } \mathrm{eV}, \\
\Gamma_{1 s} & =249 \pm 111 \text { (stat) } \pm 30 \text { (syst) } \mathrm{eV} .
\end{aligned}
$$

As can be seen from the above result, the accuracy is still tens of eV in the energy shift and more than $100 \mathrm{eV}$ in the width. Now DEAR is being followed by the SIDDHARTA experiment that will feature new silicon drift detectors. The plans of the SIDDHARTA collaboration include the measurement of both the energy shift and the width of kaonic hydrogen with a precision of several $\mathrm{eV}$, i.e. at the few percent level, by 2007. Moreover, SIDDHARTA will attempt the first ever measurement of the energy shift of the kaonic deuterium with a comparable accuracy and possibly, kaonic helium and sigmonic atoms.

The necessity to perform measurements of the kaonic deuterium ground-state observables is justified by the fact that, unlike in the case of pionic atoms, the measurement of only the kaonic hydrogen spectrum does not allow even in principle - to extract independently both S-wave $\bar{K} N$ scattering lengths $a_{0}$ and $a_{1}$. This happens because the imaginary parts of these scattering lengths do not vanish in the isospin limit, being determined by the decays into inelastic strong channels $\pi \Sigma, \pi^{0} \Lambda, \cdots$. Consequently, one attempts here to determine four independent quantities (real and imaginary parts of $a_{0}$ and $a_{1}$ ) that requires performing four independent measurements - e.g., the energy level shifts and widths of kaonic hydrogen and kaonic deuterium. However, even though it is clear that $a_{0}$ and $a_{1}$ can not be determined separately without measuring kaonic deuteron, it is still not evident whether it is possible to do so if one performs such a measurement. The reason is that the (complex) kaon-deuteron amplitude at threshold, which is directly determined from the experiment and which is expressed in terms of $a_{0}$ and $a_{1}$ through the multiple-scattering series, is generally plagued by systematic uncertainties due to a poor knowledge of the low-energy kaon-nucleon dynamics. Thus, we have to understand in advance, whether these uncertainties are small enough not to hinder a determination of $a_{0}$ and $a_{1}$ from the forthcoming SIDDHARTA experiment. This is the main purpose of the present paper.

The kaon-deuteron scattering process has been extensively studied in the past within potential (multiple scattering) approaches (see, e.g. [3-6]). To our knowledge, up to now the most detailed investigation of the problem within an effective field theory (EFT) framework is carried out in Ref. [7] on the basis of the so-called chiral unitary approach. In the present paper we undertake a new investigation of the problem within a systematic field-theoretical approach which, at the lowest order, is formally similar to that of Ref. [7]. In addition to the previous studies, in this paper we also consider different sources of the systematic 
Ulf-G. Meißner et al.: Kaon-nucleon scattering lengths from kaonic deuterium experiments

error in the calculations, and try to give - whenever possible - a crude estimate of the theoretical uncertainty.

The main difference of the present article to previous work is, however, that the existing approaches were exclusively concentrated on the prediction of the $K^{-} d$ scattering length from the input $\bar{K} N$ scattering lengths. We are not aware of the "reversed" analysis in the literature, where the $\bar{K} N$ scattering lengths are determined from the input data of kaonic hydrogen and deuterium ground-state shift and width. However, this is exactly the type of the analysis that will be required in the near future for the SIDDHARTA data. In this paper - in the absence of any experimental data - we use "synthetic" data and show that the reversed calculations, owing to the non-linear dependence of the kaon-deuteron amplitude on the $\bar{K} N$ scattering lengths, turn out to be much more sensitive to the theoretical input on the deuteron structure and the kaon-deuteron interactions, than a straightforward evaluation of the $K^{-} d$ scattering length through the multiple-scattering series. This fact could potentially render a combined analysis of the hydrogen and deuterium data a beautiful testing ground for different EFT descriptions of the low-energy kaon-deuteron interactions and, as a result, might enable one to accurately determine the values of the scattering lengths $a_{0}$ and $a_{1}$.

The paper is organized as follows. In section 2 we consider the extraction of the complex kaon-deuteron scattering length from the data on kaonic deuterium. The multiple-scattering series for the kaon-deuteron scattering amplitude is studied in section 3 and the issue of isospin breaking is addressed in section 4 . Further, in section 5 the numerical results of the simultaneous analysis of the existing kaonic hydrogen and synthetic kaonic deuterium data are presented and discussed. Section 6 contains our conclusions.

\section{Kaonic deuterium}

In the experiments on hadronic atoms one measures the energy levels and widths of this sort of bound states. At present, there exists a well established systematic procedure for extracting the values of the pertinent hadronic scattering amplitudes at threshold from these measurements, based on non-relativistic effective Lagrangians (see e.g. [8-13]). In particular, the case of the kaonic hydrogen has been addressed in Ref. [12]. The calculation of the ground state energy of kaonic deuteron is completely analogous to the derivation of the Deser-type formula in the case of pionic deuterium, which was carried out in Ref. [13] - we do not repeat this derivation here. However, as first pointed out in Ref. [14], in the case of kaonic atoms one should in addition check whether the relatively large decay widths of these atoms leads to a conflict with the use of the Rayleigh-Schrödinger perturbation expansion for the energy levels. In that article it is proposed to estimate the quantity $\tau E_{1 s}^{d}$, where $\tau$ denotes the lifetime and $E_{1 s}^{d}$ the binding energy for the ground state. Should it turn out that this product is of order one, using RayleighSchrödinger perturbation theory would be questionable.
The lifetime of kaonic deuterium is determined by the imaginary part of the $K^{-} d$ scattering length. In the absence of any reliable experimental information, we use the crude estimate $\operatorname{Im} A_{K d} \simeq 1 \mathrm{fm}$ which is consistent with the analysis of Ref. [15]. The decay width and the groundstate binding energy at lowest order in the fine-structure constant $\alpha$ are given by

$$
\begin{aligned}
& \Gamma_{1 s}^{d}=\frac{1}{\tau} \simeq 4 \alpha^{3} \mu_{r}^{2} \operatorname{Im} A_{K d} \simeq 1.2 \mathrm{keV}, \\
& E_{1 s}^{d} \simeq \frac{1}{2} \mu_{r} \alpha^{2} \simeq 10.4 \mathrm{keV} .
\end{aligned}
$$

This yields $\tau E_{1 s}^{d} \simeq 8.6$, which is still large enough to justify using perturbation theory (here, $\mu_{r}$ denotes the reduced mass of the kaon-deuteron system).

The (complex) kaon-deuteron scattering length $A_{K d}$ can be extracted from the future SIDDHARTA data by using the Deser-type formula at next-to-leading order in isospin breaking, which is the same as in Ref. [13]

$$
\begin{aligned}
\epsilon_{1 s}^{d} & -i \frac{\Gamma_{1 s}^{d}}{2}=-2 \alpha^{3} \mu_{r}^{2} A_{K d} \\
& \times\left\{1-2 \alpha \mu_{r} A_{K d}(\ln \alpha-1)+\cdots\right\},
\end{aligned}
$$

where the ellipses stand for small contributions which can be neglected at the accuracy we are working. On the other hand, since $\tau E_{1 s}^{d}$ is not very large, it is not excluded that the corrections at next-to-next-to-leading order in isospin breaking (which are expected to amount to a few percent) should also be taken into account, when the accuracy of SIDDHARTA data is close to the planned one. It is however, well-known that such calculations can be performed in a straightforward manner within the non-relativistic EFT and hence, no uncontrollable systematic error finally emerges at this place. One may therefore safely assume that the quantity $A_{K d}$ is directly determined from the experiment.

\section{Multiple-scattering series for the kaon-deuteron amplitude}

The main purpose of the present paper is to investigate whether the measurement of the quantity $A_{K d}$, along with the $K^{-} p$ elastic scattering amplitude at threshold that is separately determined in the experiment on kaonic hydrogen, enables one to extract precise values of $a_{0}$ and $a_{1}$. The first step is to express $A_{K d}$ in terms of $a_{0}$ and $a_{1}$ (and possibly, other physical parameters characterizing the low-energy $\bar{K} N$ interaction) within the multiplescattering theory. This procedure can be appropriately formulated by using the language of effective non-relativistic Lagrangians (see, e.g. $[13,16,17])$ that, in addition, allows one to systematically calculate the corrections. However, a strong predictive power of the non-relativistic approach is in fact based on a very subtle balance of different momentum scales involved in the problem [13,22]. Namely, in this approach the couplings of the effective Lagrangian 
are easily expressed in terms of the threshold parameters of the elementary $\bar{K} N$ interactions and the perturbative expansion of the kaon-deuteron scattering amplitude corresponds to the usual multiple-scattering series. In analogy with the case of the pion-deuteron system one would, however, expect that the three-body low-energy constant (LEC), which describes contact interaction between the kaon and two nucleons, is strongly enhanced and, since the value of this LEC is unknown, this leads to a large systematic error in the predicted kaon-deuteron scattering length $[13,16,17]$. On the other hand, if one uses the EFT with non-perturbative pions based on the Weinberg counting rules (see [24] for a recent review), both the dimensional analysis and resonance saturation [13], as well as the study of the scale dependence $[22,23]$ carried out in the pion-deuteron case, indicate on a rather small uncertainty due to the three-body interactions - at the cost of the fact that now the expansion of the pion-deuteron scattering length should be carried out in Chiral Perturbation Theory (ChPT) and not in terms of the threshold parameters of the underlying $\pi N$ scattering amplitudes. The latter property may lead to even more serious problems in the kaon-deuteron case, owing to the non-perturbative character of the low-energy $\bar{K} N$ interactions and the necessity of using the chiral unitary approach (see, e.g. [18-21]). The solution to the above dilemma lies in the observation of a certain hierarchy of various contributions in the theory with non-perturbative pions, which could be described by the so-called modified power counting [22]. The existence of such a hierarchy, which can be traced back to the suppression of the diagrams with the creation/annihilation of virtual pions, indicates that the structure of the theory closely resembles that of the non-relativistic theory up to the three-body contact terms, whose value has to be determined from the matching of these two theories. Further, from the direct comparison of the expressions of the piondeuteron scattering lengths calculated within these two approaches one may conclude that the lowest-order threebody LEC in the non-relativistic theory can be effectively omitted, if the deuteron wave function, calculated in the EFT with non-perturbative pions, is used to evaluate matrix elements in the non-relativistic EFT. Below, we shall use this simple prescription for estimating the value of the three-body LEC in the case of the kaon-deuteron system as well.

The pion-deuteron and kaon-deuteron systems differ in one crucial aspect. As it is well known (see, e.g. [7] and references therein), the multiple-scattering series for the kaon-deuteron scattering does not converge and requires a (partial) re-summation. This can be done most simply by using the so-called Fixed Center Approximation (FCA), in which the nucleons are considered to be infinitely heavy. The validity of this approximation has been studied both in the potential scattering theory (see e.g. [25]) and in the EFT approach [26]. The fact that FCA can be a reasonable approximation even for $M_{K} / m_{p} \simeq 0.5$ (see Ref. [7] and references therein) is related to the peculiar cancellations at second order, which are discussed in Refs. $[25,26]$ (here, $M_{K}$ and $m_{p}$ denote the masses of the charged kaon and the proton, respectively). In the secondorder calculations in the non-relativistic EFT, which we have carried out, it is indeed possible to analytically verify the cancellation of the corrections to the FCA at leading order in $M_{K} / m_{p}$ (here, one can use the corresponding analytic expressions from Ref. [13] after the substitution $\left.M_{\pi} \rightarrow M_{K}\right)$. Moreover, it should be pointed out that in this theory the "binding corrections" [25] should be also included in the second-order term and, as a result, the cancellation occurs in both isospin channels and not just in one as in Ref. [26]. Numerically, utilizing dimensional regularization, the corrections turn out to be of order of $20-30 \%$ for the kaon-deuteron system and of order of a few percent in the pion-deuteron system. We expect these numbers to further decrease if the wave functions calculated in EFT with non-perturbative pions are used in the calculations. Note that, to the best of our knowledge, there exists no proof of cancellations beyond second order, but from e.g. the comparison to the exact solution of Faddeev equations [5] (see also the discussion in Ref. [7]) one may conclude that the numbers quoted above give a realistic estimate of the theoretical error due to FCA at all orders. It should however, be stressed that the EFT approach which is used in the present paper provides an appropriate tool for a systematic calculation of the corrections to the FCA, as well as the inclusion of higher-order (derivative) interactions. In order to provide a sufficient theoretical accuracy for analyzing SIDDHARTA results, such calculations might be necessary in the future.

Using the FCA in the non-relativistic EFT and neglecting derivative interactions, we arrive at the expression for the kaon-deuteron scattering length, which is formally similar to the one from Ref. [7]:

$$
\left(1+\frac{M_{K}}{M_{d}}\right) A_{K d}=\int_{0}^{\infty} d r\left(u^{2}(r)+w^{2}(r)\right) \hat{a}_{k d}(r),
$$

where $M_{d}$ is the deuteron mass, $u(r)$ and $w(r)$ denote the usual $S$ - and $D$-wave components of the deuteron wave function, which are normalized via the condition $\int_{0}^{\infty} d r\left(u^{2}(r)+w^{2}(r)\right)=1$, and

$$
\hat{a}_{k d}(r)=\frac{\tilde{a}_{p}+\tilde{a}_{n}+\left(2 \tilde{a}_{p} \tilde{a}_{n}-b_{x}^{2}\right) / r-2 b_{x}^{2} \tilde{a}_{n} / r^{2}}{1-\tilde{a}_{p} \tilde{a}_{n} / r^{2}+b_{x}^{2} \tilde{a}_{n} / r^{3}}+\delta \hat{a}_{k d}
$$

with $b_{x}^{2}=\tilde{a}_{x}^{2} /\left(1+\tilde{a}_{u} / r\right)$. Further,

$$
\left(1+\frac{M_{K}}{m_{p}}\right) a_{p, n, x, u}=\tilde{a}_{p, n, x, u}
$$

where $a_{p, n, x, u}$ denote the threshold scattering amplitudes for $K^{-} p \rightarrow K^{-} p, K^{-} n \rightarrow K^{-} n, K^{-} p \rightarrow \bar{K}^{0} n$ and $\bar{K}^{0} n \rightarrow \bar{K}^{0} n$, respectively. Finally, the quantity $\delta \hat{a}_{k d}$ is proportional to the three-body LEC $f_{0}^{K}$, which is a counterpart of the quantity $f_{0}$ introduced in Ref. [13]. As discussed above, one may assume that $f_{0}^{K}$ vanishes, if the deuteron wave functions $u(r)$ and $w(r)$ are those calculated in the EFT with non-perturbative pions. We have 
Ulf-G. Meißner et al.: Kaon-nucleon scattering lengths from kaonic deuterium experiments

further carried out the dimensional estimate of the resulting uncertainty, which yields a few percent theoretical error in the kaon-deuteron scattering length. Thus, the whole procedure is consistent.

The calculations with the deuteron wave functions of the non-relativistic EFT, which at the leading order are given by

$$
u(r)=\sqrt{2 \gamma} \mathrm{e}^{-\gamma r}, \quad w(r)=0, \quad \gamma^{2} \simeq m_{p} E_{d},
$$

are more subtle (here $E_{d}$ denotes the binding energy of the deuteron). Namely, expanding Eq. (5) in the multiplescattering series corresponding to an expansion in powers of $1 / r$ and integrating this term-by-term, it is immediately seen that the subsequent terms diverge worse and worse as $r \rightarrow 0$. This means that the pertinent integrals require renormalization and are scale-dependent (see e.g. Refs. $[13,16,17]$ ). On the other hand, the integral before such an expansion is well-defined and does not have any scale dependence. It is however, clear that the uncertainty which is related to the existence of (large) three-body contact interactions can not simply disappear as a result of the re-summation of the multiple-scattering series. To solve this puzzle, note that the non-relativistic theory makes sense only for the momenta $|\mathbf{p}| \ll \lambda$, with $\lambda$ being of the order of the pion mass. Hence, in order to effectively mimic the effect of the short-range physics, one could straightforwardly perform cutoff regularization of the multiple-scattering series and study the cutoff dependence of the calculated kaon-deuteron scattering length after the re-summation of the series (even now the limit $\lambda \rightarrow \infty$ exists). This can be done most easily through the replacement $1 / r \rightarrow\{1-\exp (-\lambda r)\} / r$ in Eq. (5). Further, in order to relate the value of $\lambda$ to the scale $\mu$ of dimensional regularization, which is usually used in the non-relativistic EFT, we calculate the mean value of the operator $1 / r$ between the wave functions given by Eq. (7) twice: once using dimensional regularization with minimal subtraction and once using cutoff regularization. The comparison of these two results gives $\lambda=\sqrt{e} \mu \simeq 1.65 \mu$ (where $e$ denotes the basis of natural logarithm), so that the interval $100 \mathrm{MeV} \leq \mu \leq 250 \mathrm{MeV}$, which was fixed in Ref. [13], is mapped onto the interval $165 \mathrm{MeV} \leq \lambda \leq 412 \mathrm{MeV}$. Note also that, if instead of the wave functions Eq. (7) the wave functions from the EFT with non-perturbative pions are used, the calculated kaon-deuteron scattering length has practically no $\lambda$-dependence for $165 \mathrm{MeV} \leq \lambda \leq \infty$. This shows that it is consistent to use an "unregularized" $1 / r$ operator together with these wave functions, because the necessary cutoff is provided by the wave function itself.

\section{Isospin breaking}

The equation (5) contains four different combinations of the threshold amplitudes. Consequently, one has first to relate these amplitudes to the two scattering lengths $a_{0}$ and $a_{1}$, which should then be determined from the analysis of the combined data on kaonic hydrogen and deuterium. In this work we take into account the leading-order
Table 1. $\bar{K} N$ scattering lengths $a_{0}$ and $a_{1}$ (in fm) from the literature. These scattering lengths are used as an input in the calculations of the kaon-deuteron scattering length.

\begin{tabular}{|l|l|l|}
\hline Ref. & $a_{0}$ & $a_{1}$ \\
\hline Meißner and Oller [19] & $-1.31+i 1.24$ & $0.26+i 0.66$ \\
\hline Borasoy et al., fit u [20] & $-1.48+i 0.86$ & $0.57+i 0.83$ \\
\hline Oller et al., fit A4 [21] & $-1.23+i 0.45$ & $0.98+i 0.35$ \\
\hline Martin [27] & $-1.70+i 0.68$ & $0.37+i 0.60$ \\
\hline
\end{tabular}

isospin-breaking corrections in the kaon-nucleon scattering amplitudes which are due to the unitary cusps [12]. The re-summation of the bubble diagrams leads to the following simple parameterization

$$
\begin{aligned}
& a_{p}=\frac{\frac{1}{2}\left(a_{0}+a_{1}\right)+q_{0} a_{0} a_{1}}{1+\frac{q_{0}}{2}\left(a_{0}+a_{1}\right)}, \quad a_{n}=a_{1}, \\
& a_{x}=\frac{\frac{1}{2}\left(a_{0}-a_{1}\right)}{1-\frac{i q_{c}}{2}\left(a_{0}+a_{1}\right)}, \quad a_{u}=\frac{\frac{1}{2}\left(a_{0}+a_{1}\right)-i q_{c} a_{0} a_{1}}{1-\frac{i q_{c}}{2}\left(a_{0}+a_{1}\right)},
\end{aligned}
$$

where

$$
\begin{aligned}
& q_{c}=\sqrt{2 \mu_{c} \Delta}, \quad q_{0}=\sqrt{2 \mu_{0} \Delta}, \\
& \Delta=m_{n}+M_{\bar{K}^{0}}-m_{p}-M_{K}, \\
& \mu_{c}=\frac{m_{p} M_{K}}{m_{p}+M_{K}}, \quad \mu_{0}=\frac{m_{n} M_{\bar{K}^{0}}}{m_{n}+M_{\bar{K}^{0}}},
\end{aligned}
$$

with $m_{n}, M_{\bar{K}^{0}}$ being the masses of the neutron and the $\bar{K}^{0}$, respectively. Further, in the calculations we have used the input scattering lengths evaluated within various versions of the so-called chiral unitary approach [19-21], as well as the experimental values from Ref. [27]. Table 1 collects these input values. Note that for us it is not possible to straightforwardly use the scattering lengths (isospin basis) from Refs. [7, 18], since the definition of these scattering lengths (in the isospin limit) differs from the one, which is used here or in Refs. [19-21]. In particular, in Refs. [7,18] $a_{0}, a_{1}$ are determined from the $\bar{K} N$ scattering amplitude with CM energy taken at $K^{-} p$ threshold, whereas the masses of all particles in the isospin limit are taken equal to the average masses in the isospin multiplets $^{1}$. Note that, in contrary to this, the usual definition of the scattering lengths in the isospin limit of QCD plus QED implies that the CM energy is set exactly equal to the threshold energy.

\section{Numerical results and discussion}

The tables 2, 3 and 4 contain our results of the calculations of the kaon-deuteron scattering length with the use of the

\footnotetext{
1 We are thankful to E. Oset and J. Oller for a clarifying discussion on this and other related topics.
} 
above formulae. In particular, in table 2 the results obtained by using the leading-order deuteron wave function in the non-relativistic EFT (see Eq. (7)) are displayed for different values of the cutoff parameter $\lambda$ (or, equivalently, $\mu$ ). Table 3 contains the results obtained by using the Paris and Bonn potential model wave functions as well as the deuteron wave functions calculated at NLO in the EFT with non-perturbative pions for two different values of the cutoff parameter $\Lambda$, which is present in this theory. From table 2 one sees - as expected from Refs. [13,16,17] - a rather strong dependence of the calculated kaon-deuteron scattering length on the cutoff parameter $\lambda$, which has not disappeared after carrying out the re-summation of the multiple-scattering series. On the other hand, the dependence on the parameter $\Lambda$ in table 3 is very weak (cf. with $[22,23])$ and all wave functions in this table yield practically the same result, which in most cases - except for the input from Ref. [21] - coincides with the result of table 2 somewhere around $\mu=M_{\pi}$. Consequently, this yields an useful "rule of the thumb" for a rough estimate of the value of the three-body LEC $f_{0}^{K}$ in the non-relativistic EFT.

Finally, in table 4 we show the effect of the isospin breaking on the theoretical value of the kaon-deuteron scattering length. Bearing in mind the large isospin-breaking corrections in the $\bar{K} N$ amplitudes [12] as well as in the pionic deuterium [28], it is a little surprising that in most cases - again except the input from Ref. [21] - the leading-order isospin-breaking effect at the end turns out to be very small. Note also that the large isospin-breaking corrections, which are quoted in Ref. [7], most probably result from the peculiar definition of the scattering lengths in the isospin limit, which is adopted there.

Next, we turn to the main goal of the present paper: how does one extract precise data of the kaon-nucleon scattering lengths $a_{0}$ and $a_{1}$ from the combined measurements of the (complex) ground-state energy in the kaonic hydrogen and kaonic deuterium, which are carried out by the DEAR/SIDDHARTA collaboration? Let us start from the kaonic hydrogen case, for which the preliminary results of the measurements are already known. These measurements alone do not suffice to determine the values of both $a_{0}$ and $a_{1}$, but yield a relation between these two quantities that leads to a strong restriction on the possible values that $a_{0}$ and $a_{1}$ can take. In order to understand how this restriction emerges, let us consider the expression for the ground-state energy of the kaonic hydrogen at next-to-leading order in isospin breaking [12]

$$
\epsilon_{1 s}-\frac{i}{2} \Gamma_{1 s}=-2 \alpha^{3} \mu_{c}^{2} a_{p}\left\{1-2 \alpha \mu_{c}(\ln \alpha-1) a_{p}\right\}
$$

With the help of this equation one may directly extract the quantity $a_{p}$ from the DEAR data. Using now Eq. (8) to relate the quantities $a_{p}$ and $a_{0}, a_{1}$ we obtain

$$
a_{0}+a_{1}+\frac{2 q_{0}}{1-q_{0} a_{p}} a_{0} a_{1}-\frac{2 a_{p}}{1-q_{0} a_{p}}=0 .
$$

Together with the requirement $\operatorname{Im} a_{I} \geq 0$, which stems from unitarity, Eq. (11) defines a circle in the $\left(\operatorname{Re} a_{I}\right.$,

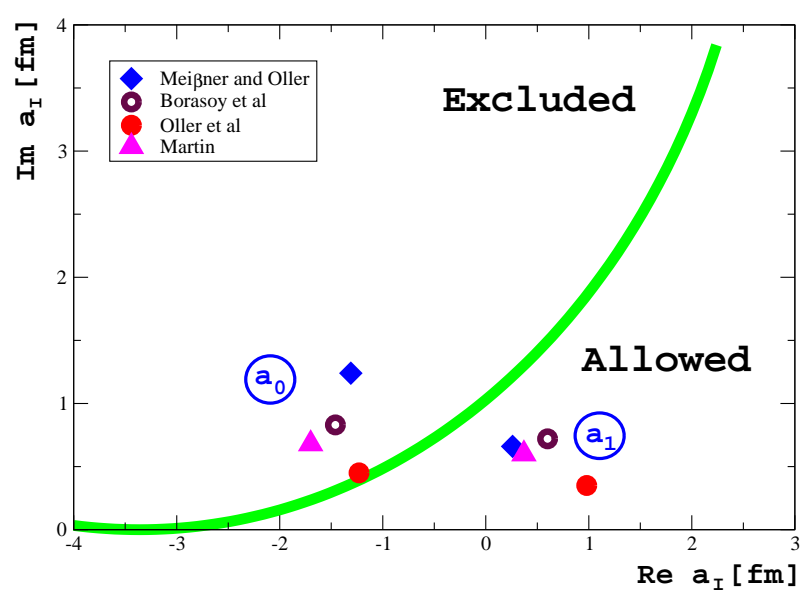

Fig. 1. Restrictions set by the DEAR data on the values of the scattering lengths $a_{0}$ and $a_{1}$. For comparison, we give the scattering length calculations from different analyses: 1) Meißner and Oller [19], 2) Borasoy, Nißler and Weise, fit u [20], 3) Oller, Prades and Verbeni, fit A4 [21], 4) Martin [27].

$\operatorname{Im} a_{I}$ )-plane. Part of this circle is shown in Fig. 1 (note that, bearing in mind the preliminary character of the DEAR data [1], we use only central values in order to illustrate the construction of the plot and do not provide a full error analysis). In order to be consistent with the DEAR data, both $a_{0}$ and $a_{1}$ should be on the right of this universal DEAR circle. For comparison, on the same figure we plot $a_{0}$ and $a_{1}$ from table 1 . As we see, in most of the approaches it is rather problematic to get a value for $a_{0}$ which is compatible with DEAR. This kind of analysis may prove useful in the near future, when the accuracy of the DEAR is increased that might stir efforts on the theoretical side, aimed at a systematic quantitative description of the $\bar{K} N$ interactions within the unitarized ChPT.

Further, from Eq. (11) one may determine e.g. $a_{1}$. Substituting this expression into Eqs. (4), (5) and (8), one arrives at a non-linear equation for determining $a_{0}$ with a given input value of $A_{K d}$. In the absence of experimental data on kaonic deuterium, we adopt the following strategy to solve this equation. In the literature, we find different theoretical calculations of the $K d$ scattering length. We consider some of them, namely the results of Refs. $[4,5,29,30]$, as synthetic experimental data and solve the corresponding equation with respect to $a_{0}$. For completeness, we also present the results which are obtained from the same synthetic deuteron data together with the old $\mathrm{KpX}$ data on kaonic hydrogen, $\epsilon_{1 \mathrm{~s}}=323 \mathrm{eV}$, $\Gamma_{1 s}=407 \mathrm{eV}[2]$. Note also that our solution includes only central values of the input kaon-deuteron and kaon-proton amplitudes. At this preliminary stage it is natural to postpone the full error analysis, until the SIDDHARTA data on the kaonic deuterium become available.

We start with the deuteron wave function in the nonrelativistic EFT, which is given by Eq. (7). The results for the extracted scattering lengths $a_{0}$ and $a_{1}$ are given 
Table 2. Kaon-deuteron scattering length $A_{K d}$ (in fm), calculated by using Eqs. $(4,5)$ (setting $\left.\delta \hat{a}_{K d}=0\right)$ and the input from table 1. The deuteron is described by the wave function in Eq. (7). The values of the regularization parameter $\lambda$ shown in this table, correspond to the following values of the dimensional regularization scale: $\mu=100 \mathrm{MeV} ; \mu=M_{\pi} ; \mu=250 \mathrm{MeV} ; \mu=\infty$ (cf. with Ref. [13]).

\begin{tabular}{|c|c|c|c|c|}
\hline Ref. & $\lambda=165 \mathrm{MeV}$ & $\lambda=231 \mathrm{MeV}$ & $\lambda=412 \mathrm{MeV}$ & $\lambda=\infty$ \\
\hline$[19]$ & $-1.35+i 1.58$ & $-1.31+i 1.42$ & $-1.21+i 1.24$ & $-1.07+i 1.13$ \\
\hline$[20]$ & $-1.63+i 1.39$ & $-1.56+i 1.24$ & $-1.41+i 1.08$ & $-1.25+i 0.99$ \\
\hline$[21]$ & $-1.30+i 1.08$ & $-1.30+i 0.96$ & $-1.18+i 0.82$ & $-1.01+i 0.75$ \\
\hline$[27]$ & $-1.70+i 1.26$ & $-1.61+i 1.14$ & $-1.46+i 0.99$ & $-1.31+i 0.90$ \\
\hline
\end{tabular}

Table 3. The same as in table 2, but with the deuteron wave functions calculated in the Paris and Bonn potential models as well as at NLO in EFT with non-perturbative pions for two different values of the cutoff parameter: NLO(1) stands for $\Lambda=450 \mathrm{MeV}$ and $\mathrm{NLO}(2)$ for $\Lambda=600 \mathrm{MeV}$. The parameter $\lambda$ is set to $\infty$.

\begin{tabular}{|c|c|c|c|c|}
\hline Ref. & Paris & Bonn & NLO $(1)$ & NLO $(2)$ \\
\hline$[19]$ & $-1.29+i 1.66$ & $-1.28+i 1.65$ & $-1.29+i 1.66$ & $-1.30+i 1.67$ \\
\hline$[20]$ & $-1.55+i 1.48$ & $-1.54+i 1.47$ & $-1.55+i 1.48$ & $-1.56+i 1.49$ \\
\hline$[21]$ & $-1.10+i 1.10$ & $-1.10+i 1.09$ & $-1.09+i 1.10$ & $-1.11+i 1.11$ \\
\hline$[27]$ & $-1.66+i 1.28$ & $-1.65+i 1.27$ & $-1.66+i 1.28$ & $-1.67+i 1.29$ \\
\hline
\end{tabular}

Table 4. Isospin-breaking corrections to the $K d$ scattering length for different input values of $a_{0}$ and $a_{1}$. NLO EFT wave functions are used for the deuteron. The label "sym" refers to the scattering length evaluated in the isospin symmetry limit.

\begin{tabular}{|c|c|c|c|c|}
\hline Ref. & $\mathrm{NLO}(1), \mathrm{sym}$ & $\mathrm{NLO}(1)$ & $\mathrm{NLO}(2), \mathrm{sym}$ & $\mathrm{NLO}(2)$ \\
\hline$[19]$ & $-1.26+i 1.65$ & $-1.29+i 1.66$ & $-1.27+i 1.66$ & $-1.30+i 1.67$ \\
\hline$[20]$ & $-1.48+i 1.46$ & $-1.55+i 1.48$ & $-1.50+i 1.47$ & $-1.56+i 1.49$ \\
\hline$[21]$ & $-0.85+i 1.00$ & $-1.09+i 1.10$ & $-0.87+i 1.01$ & $-1.11+i 1.11$ \\
\hline$[27]$ & $-1.64+i 1.22$ & $-1.66+i 1.28$ & $-1.66+i 1.24$ & $-1.67+i 1.29$ \\
\hline
\end{tabular}

in table 5, where we had to choose a rather small value of the cutoff parameter $\lambda=132 \mathrm{MeV}$ corresponding to $\mu=80 \mathrm{MeV}$. From this table one already sees the main property of the solutions: due to a highly non-linear form of the equation which determines $a_{0}$ and $a_{1}$, the solutions do not always exist (at least for those values of these scattering lengths which can be termed physically reasonable). Note that this mainly concerns the solutions which are obtained using DEAR input. The KpX data turn out to be much less restrictive. For example, if we increase the value of the parameter $\mu$ even up to $100 \mathrm{MeV}$, the sole solution with DEAR input in table 5 disappears, whereas the solutions with $\mathrm{KpX}$ input are still present. Note also that we have analyzed more synthetic input than is finally shown in table 5: for most of the input values of the kaon-deuteron scattering length which are known in the literature, there exists no solution for $a_{0}$ and $a_{1}$.

Bearing in mind the results in table 5, it is not surprising that, using the wave functions calculated an NLO in the theory with non-perturbative pions, the solutions exist only with the $\mathrm{KpX}$ input. The corresponding results are shown in table 6 .

One might finally ask the following question: how large should the kaon-deuteron scattering length be so that a solution for $a_{0}$ and $a_{1}$ exists at all? In order to answer this question, we have scanned the $\left(\operatorname{Re} A_{K d}, \operatorname{Im} A_{K d}\right)_{-}^{-}$ plane in the interval $-2 \mathrm{fm}<\operatorname{Re} A_{K d}<0$ and $0.5 \mathrm{fm}<$ $\operatorname{Im} A_{K d}<2.5 \mathrm{fm}$ and tried to find solutions, using DEAR input data. The results of this investigation, which are displayed in Fig. 2, are very interesting: it turns out that the solutions exist only if $\operatorname{Im} A_{K d} \lesssim 1 \mathrm{fm}$ and moreover, if $\operatorname{Im} A_{K d} \simeq 1 \mathrm{fm}$ then one finds solutions only in a very small interval around $\operatorname{Re} A_{K d} \simeq-1 \mathrm{fm}$. Some representative solutions are shown in table 7 . We wish to also note that all this agrees with the scattering data analysis, carried out in Ref. [15]. If $\operatorname{Im} A_{K d}$ crosses the border of the shaded area in Fig. 2 continuously from below, then on the same branch one gets the solution with $\operatorname{Im} a_{1} \leq 0$ that is 
Table 5. The scattering lengths $a_{0}$ and $a_{1}$, obtained from a simultaneous analysis of DEAR (KpX) data and the synthetic input for the $K d$ scattering length. The deuteron wave function is described by Eq. (7) and the regularization parameter $\lambda=132 \mathrm{MeV}$.

\begin{tabular}{|c|l|l|}
\hline \multicolumn{1}{|c|}{ Input $A_{K d}$} & DEAR & $\mathrm{KpX}$ \\
\hline$-1.34+i 1.04[4]$ & $\begin{array}{l}a_{0}=-1.27+i 0.36 \\
a_{1}=1.19+i 0.09\end{array}$ & $\begin{array}{l}a_{0}=-1.34+i 0.63 \\
a_{1}=0.01+i 0.55\end{array}$ \\
\hline$-0.85+i 1.10[5]$, Faddeev Eq. & - & $a_{0}=-1.40+i 0.82$ \\
& & $a_{1}=-0.09+i 0.07$ \\
\hline$-0.75+i 1.12[5]$, FCA & - & $a_{0}=-1.41+i 0.84$ \\
& & $a_{1}=-0.10+i 0.01$ \\
\hline$-0.78+i 1.23[29]$ & - & $a_{0}=-1.43+i 0.82$ \\
& & $a_{1}=-0.04+i 0.01$ \\
\hline$-1.92+i 1.58[30]$ & - & $a_{0}=-1.64+i 0.63$ \\
& & $a_{1}=1.03+i 0.02$ \\
\hline
\end{tabular}

Table 6. The same as in table 5, but with the NLO EFT wave functions and $\lambda=\infty$. Only KpX input is displayed, since there are no solutions with DEAR input.

\begin{tabular}{|c|l|l|}
\hline Input $A_{K d}$ & $\Lambda=450 \mathrm{MeV}$ & $\Lambda=600 \mathrm{MeV}$ \\
\hline$-1.34+i 1.04[4]$ & $a_{0}=-1.36+i 0.59$ & $a_{0}=-1.35+i 0.60$ \\
& $a_{1}=0.10+i 0.63$ & $a_{1}=0.07+i 0.62$ \\
\hline$-0.85+i 1.10[5]$, Faddeev Eq. & $a_{0}=-1.41+i 0.80$ & $a_{0}=-1.41+i 0.80$ \\
& $a_{1}=-0.04+i 0.08$ & $a_{1}=-0.05+i 0.08$ \\
\hline$-0.75+i 1.12[5], \mathrm{FCA}$ & $a_{0}=-1.42+i 0.82$ & $a_{0}=-1.42+i 0.82$ \\
& $a_{1}=-0.04+i 0.03$ & $a_{1}=-0.05+i 0.03$ \\
\hline$-0.78+i 1.23[29]$ & $a_{0}=-1.44+i 0.80$ & $a_{0}=-1.44+i 0.80$ \\
& $a_{1}=0.01+i 0.03$ & $a_{1}=0.00+i 0.03$ \\
\hline
\end{tabular}

forbidden by unitarity. On the other hand, if $\mathrm{KpX}$ input data are used, the solutions exist in a much larger area.

\section{Conclusions}

The message of our investigation is very clear. Up to now, in the theoretical description of the deuteron we have restricted ourselves to the lowest-order expression in the non-relativistic EFT (derivative interactions are neglected) and, in addition, worked in the limit of infinitely heavy nucleons (FCA). It is widely believed that these approximations represent a good starting point for the description of the kaon-deuteron scattering length. Moreover, in the non-relativistic EFT the corrections are systematically calculable.

Within the approximations described above it turns out that the combined analysis of DEAR/SIDDHARTA data on kaonic hydrogen and deuterium is more restric- tive than one would a priori expect. In particular, we see that solutions exist only in a rather small area of the $\left(\operatorname{Re} A_{K d}, \operatorname{Im} A_{K d}\right)$-plane. Due to this fact, in certain cases it might be possible to pin down the values of $a_{0}$ and $a_{1}$ at a reasonable accuracy, even if $A_{K d}$ itself is not measured very accurately. Moreover, if the corrections to the lowestorder approximate result, as expected, are moderate, they should not change the qualitative picture. In our opinion, it could prove very useful to perform the calculations of these corrections before starting to analyze the forthcoming SIDDHARTA data since, as one may conclude from the discussion in the present paper, exactly these corrections constitute the largest potential source of theoretical uncertainty at present. On the other hand, crude estimates show that the uncertainty, coming from the shortrange QCD dynamics (three-body LEC) is rather small and should not likely hinder the analysis of the future SIDDHARTA data on the kaonic deuteron. Note also that the above conclusion concerns DEAR input only. The analysis 


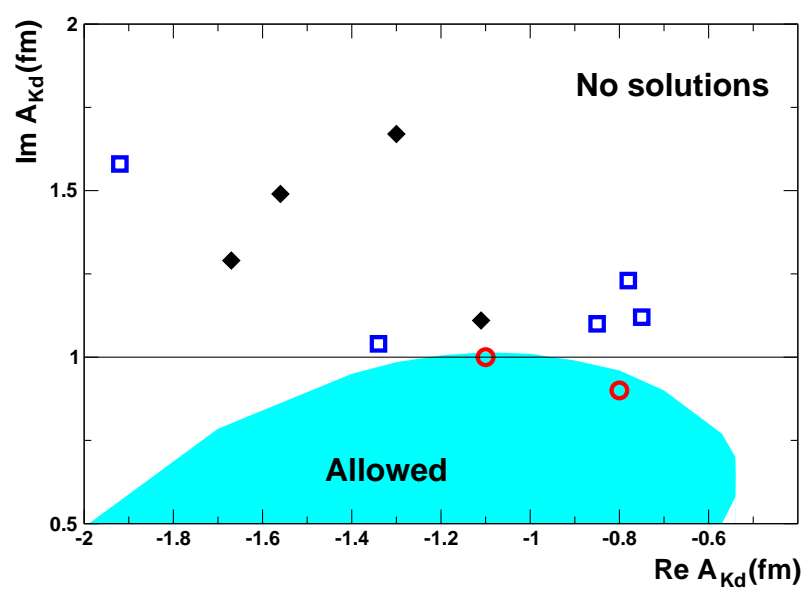

Fig. 2. The region in the $\left(\operatorname{Re} A_{K d}, \operatorname{Im} A_{K d}\right)$-plane where solutions for $a_{0}$ and $a_{1}$ exists. The cutoff parameter is $\Lambda=$ $600 \mathrm{MeV}$. For comparison, we also show the results of calculations of the kaon-deuteron scattering length from table 3 (filled diamonds), the synthetic data from tables 5, 6 (squares) and our representative solutions from table 7 (circles).

Table 7. Representative solutions for $a_{0}$ and $a_{1}$ using the NLO EFT wave functions together with DEAR input and $\operatorname{Re} A_{K d} \simeq$ $-1 \mathrm{fm}, \operatorname{Im} A_{K d} \simeq 1 \mathrm{fm}$.

\begin{tabular}{|c|l|l|}
\hline$A_{K d}$ & $\Lambda=450 \mathrm{MeV}$ & $\Lambda=600 \mathrm{MeV}$ \\
\hline$-1.10+i 1.00$ & $a_{0}=-1.25+i 0.38$ & $a_{0}=-1.24+i 0.38$ \\
& $a_{1}=1.06+i 0.00$ & $a_{1}=1.02+i 0.03$ \\
\hline$-0.80+i 0.90$ & $a_{0}=-1.07+i 0.43$ & $a_{0}=-1.06+i 0.42$ \\
& $a_{1}=0.44+i 0.07$ & $a_{1}=0.42+i 0.08$ \\
\hline
\end{tabular}

using $\mathrm{KpX}$ input turns out to be much less restrictive and therefore less informative than with the DEAR input.

To summarize, one may expect that the combined analysis of the forthcoming high-precision data from DEAR/SIDDHARTA collaboration on kaonic hydrogen and deuterium will enable one to perform a stringent test of the framework used to describe low-energy kaondeuteron scattering, as well as to extract the values of $a_{0}$ and $a_{1}$ with a reasonable accuracy. However, in order to do so, much theoretical work related to the systematic calculation of higher-order corrections within the non-relativistic EFT is still to be carried out.

Acknowledgments: The authors would like to thank Jürg Gasser, Christoph Hanhart, Vadim Lensky, Andreas Nogga and Sasha Sibirtsev for discussions. We in addition thank Evgeny Epelbaum for providing the values of the NLO wave function of the deuteron and Robin Nißler for communicating the separate values of $a_{0}$ and $a_{1}$ in their approach to us. Partial financial support from the EU Integrated Infrastructure Initiative Hadron
Physics Project (contract number RII3-CT-2004-506078) and DFG (SFB/TR 16, "Subnuclear Structure of Matter") is gratefully acknowledged.

\section{References}

1. G. Beer et al. [DEAR Collaboration], Phys. Rev. Lett. 94 (2005) 212302.

2. M. Iwasaki et al., Phys. Rev. Lett. 78 (1997) 3067.

3. J. H. Hetherington and L. H. Schick, Phys. Rev. 137 (1965) B935.

4. M. Torres, R. H. Dalitz and A. Deloff, Phys. Lett. B 174 (1986) 213.

5. A. Deloff, Phys. Rev. C 61 (2000) 024004.

6. R. C. Barrett and A. Deloff, Phys. Rev. C 60 (1999) 025201.

7. S. S. Kamalov, E. Oset and A. Ramos, Nucl. Phys. A 690, 494 (2001) [arXiv:nucl-th/0010054].

8. P. Labelle and K. Buckley, arXiv:hep-ph/9804201; X. Kong and F. Ravndal, Phys. Rev. D 59 (1999) 014031; Phys. Rev. D 61 (2000) 077506 [arXiv:hep-ph/9905539]; B. R. Holstein, Phys. Rev. D 60 (1999) 114030 [arXiv:nuclth/9901041]; D. Eiras and J. Soto, Phys. Rev. D 61 (2000) 114027 [arXiv:hep-ph/9905543].

9. A. Gall, J. Gasser, V. E. Lyubovitskij and A. Rusetsky, Phys. Lett. B 462 (1999) 335 [arXiv:hep-ph/9905309]; Phys. Rev. D 64 (2001) 016008 [arXiv:hep-ph/0103157]; J. Gasser, V. E. Lyubovitskij and A. Rusetsky, Phys. Lett. B 471 (1999) 244 [arXiv:hep-ph/9910438];

10. J. Schweizer, Phys. Lett. B 587 (2004) 33 [arXiv:hepph/0401048]; Eur. Phys. J. C 36 (2004) 483 [arXiv:hepph/0405034].

11. V. E. Lyubovitskij and A. Rusetsky, Phys. Lett. B 494 (2000) 9 [arXiv:hep-ph/0009206]; J. Gasser, M. A. Ivanov, E. Lipartia, M. Mojžiš and A. Rusetsky, Eur. Phys. J. C 26 (2002) 13 [arXiv:hep-ph/0206068].

12. U.-G. Meißner, U. Raha and A. Rusetsky, Eur. Phys. J. C 35 (2004) 349 [arXiv:hep-ph/0402261].

13. U.-G. Meißner, U. Raha and A. Rusetsky, Eur. Phys. J. C 41 (2005) 213 [arXiv:nucl-th/0501073].

14. J. Gasser, arXiv:hep-ph/0412393.

15. A. Sibirtsev, M. Büscher, V. Y. Grishina, C. Hanhart, L. A. Kondratyuk, S. Krewald and U.-G. Meißner, Phys. Lett. B 601 (2004) 132 [arXiv:nucl-th/0406061].

16. B. Borasoy and H. W. Grießhammer, Int. J. Mod. Phys. E 12 (2003) 65 [arXiv:nucl-th/0105048].

17. S. R. Beane and M. J. Savage, Nucl. Phys. A 717 (2003) 104 [arXiv:nucl-th/0204046].

18. E. Oset and A. Ramos, Nucl. Phys. A 635 (1998) 99 [arXiv:nucl-th/9711022].

19. J. A. Oller and U.-G. Meißner, Phys. Lett. B 500 (2001) 263 [arXiv:hep-ph/0011146].

20. B. Borasoy, R. Nißler and W. Weise, Phys. Rev. Lett. 94 (2005) 213401 [arXiv:hep-ph/0410305]; Eur. Phys. J. A 25 (2005) 79 [arXiv:hep-ph/0505239].

21. J. A. Oller, J. Prades and M. Verbeni, Phys. Rev. Lett. 95 (2005) 172502 [arXiv:hep-ph/0508081].

22. S. R. Beane, V. Bernard, E. Epelbaum, U.-G. Meißner and D. R. Phillips, Nucl. Phys. A 720 (2003) 399 [arXiv:hepph/0206219].

23. A. Nogga and C. Hanhart, Phys. Lett. B 634 (2006) 210 [arXiv:nucl-th/0511011]. 
24. E. Epelbaum, arXiv:nucl-th/0509032, Prog. Part. Nucl. Phys. (2006) in print.

25. G. Fäldt, Phys. Scripta 16 (1977) 81.

26. V. Baru, C. Hanhart, A. E. Kudryavtsev and U.G. Meißner, Phys. Lett. B 589 (2004) 118 [arXiv:nuclth/0402027].

27. A. D. Martin, Nucl. Phys. B 179 (1981) 33.

28. U.-G. Meißner, U. Raha and A. Rusetsky, arXiv:nuclth/0512035.

29. V. Y. Grishina, L. A. Kondratyuk, M. Büscher and W. Cassing, Eur. Phys. J. A 21 (2004) 507 [arXiv:nuclth/0402093].

30. A. Bahaoui, C. Fayard, T. Mizutani and B. Saghai, Phys. Rev. C 68, 064001 (2003) [arXiv:nucl-th/0307067]. 